\title{
PD-L1 Promotes Self-Renewal and Tumorigenicity of Malignant Melanoma Initiating Cells
}

\author{
Fang Zheng, ${ }^{1}$ Jianzhong Dang, ${ }^{2}$ Hui Zha, ${ }^{1}$ Bingyu Zhang, ${ }^{1}$ Ming Lin, ${ }^{1}$ and Fanjun Cheng ${ }^{3}$ \\ ${ }^{1}$ Department of Pediatrics, Union Hospital, Tongji Medical College, Huazhong University of Science and Technology, \\ Wuhan 430022, China \\ ${ }^{2}$ Department of Geriatrics, Renmin Hospital of Wuhan University, Wuhan 430020, China \\ ${ }^{3}$ Department of Hematology, Union Hospital, Tongji Medical College, Huazhong University of Science and Technology, Wu Han, China
}

Correspondence should be addressed to Fanjun Cheng; chengfanjun001@yeah.net

Received 22 April 2017; Accepted 10 September 2017; Published 9 November 2017

Academic Editor: Ilary Ruscito

Copyright (C) 2017 Fang Zheng et al. This is an open access article distributed under the Creative Commons Attribution License, which permits unrestricted use, distribution, and reproduction in any medium, provided the original work is properly cited.

\begin{abstract}
Recent studies have indicated that therapeutic antibodies targeting PD-L1 show remarkable efficacy in clinical trials in multiple tumors and that a melanoma cell-intrinsic PD-1: PD-L1 axis promotes tumor growth. However, few studies have shown tumorintrinsic PD-L1 effects in malignant melanoma initiating cells (MMICs). Here, we aim to determine the possible regulatory effects of PD-L1 on MMICs. The ALDEFLUOR kit was used to identify ALDH ${ }^{+}$MMICs. Flow cytometry was used to examine the expression of PD-L1 on ALDH ${ }^{+}$MMICs. To determine the role of PD-L1 in MMICs self-renewal, we cultured melanoma cells with anti-PD-L1 and measured tumorsphere formation and apoptosis. In addition, the effects of anti-PD-L1 on tumorigenicity and residual $\mathrm{ALDH}^{+}$

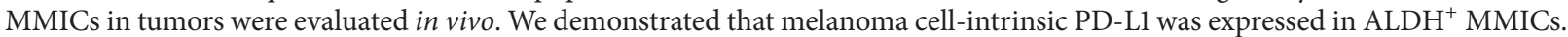
Blocking PD-L1 in melanoma cell lines impaired tumorsphere formation and induced the apoptosis of sphere cells. In addition, blocking PD-L1 inhibited tumor growth in vivo. We observed residual $\mathrm{ALDH}^{+} \mathrm{MMICs}$ within the tumor. The results showed that blocking PD-L1 also significantly decreased the residual ALDH ${ }^{+}$MMICs in the tumors. In conclusion, these results suggest a new mechanism underlying melanoma progression and PD-L1-targeted therapy, which is distinct from the immunomodulatory actions of PD-L1.
\end{abstract}

\section{Introduction}

Metastatic melanoma is an extraordinarily challenging cancer, with a 16\% 5-year survival rate, and it responds poorly to most standard chemotherapies [1]. It has been established that malignant melanoma initiating cells (MMICs) possess not only the capacity for self-renewal, differentiation, immune evasion, and multidrug resistance, but also potentially vasculogenic mimicry and the ability to transition to migratory and metastasizing derivatives, which are associated with melanoma progression and metastasis $[2,3]$. For this reason, melanoma cure is predicated upon effectively targeting and eradicating the MMICs.

Recently, it has been established that programmed death1 (PD-1) is a prominent checkpoint receptor that, upon binding its ligands $\mathrm{PD}-\mathrm{L} 1$ or $\mathrm{PD}-\mathrm{L} 2$, dampens T effector functions by inhibiting signaling downstream of the $\mathrm{T}$ cell receptor [4].
PD-L2 is predominantly expressed in APCs, whereas PDL1 is commonly expressed in various cell types, including tumor cells, immune cells, epithelial cells, and endothelial cells $[1,5]$. When PD-1 binds to its ligands in tumors; it leads to T-cell anergy and blocks productive antitumor immune response [6]. The first monoclonal antibody directed at PD-1, Nivolumab, was approved for treating patients with unresectable melanoma in July 2014. The other PD-1 and PDL1 directed agents are currently in Phase I-III clinical trials in multiple tumor types [7].

In contrast to the immunosuppressive effect of PD-L1, it is also known to contribute to the promotion of tumor cell growth and downregulation of quiescent cells $[8,9]$. Furthermore, it has been found that glioma stem cells express lower levels of PD-L1 than differentiated glioma cells do [10]. In head and neck squamous cell carcinoma, PD-L1 is preferentially expressed in $\mathrm{CD} 44^{+}$tumor-initiating cells 
[11]. PD-L1 also has suppressive effects on cancer stem cellrelated phenotypes of cholangiocarcinoma [12]. These recent data highlight the possible involvement of PD-L1 in the regulation of cancer stem cells in various tumors. However, little research has investigated the role of PD-L1 in MMICs. Here, we report on a study to determine the frequency of PDL1 expression in MMICs, and the possible regulatory effects of PD-L1 on MMICs.

\section{Materials and Methods}

2.1. Cells and Cell Culture. B16-F0 and B16-F1 melanoma cell lines were maintained in RPMI 1640 (Gibco) containing 10\% fetal bovine serum (FBS; ScienceCell), $100 \mathrm{U} / \mathrm{ml}$ penicillin (Gibco), and $100 \mu \mathrm{g} / \mathrm{mL}$ streptomycin (Gibco). Cells were cultured at $37^{\circ} \mathrm{C}$ in $95 \%$ humidity and $5 \% \mathrm{CO}_{2}$ atmosphere. All cell lines were routinely screened for mycoplasma contamination.

2.2. Flow Cytometry. The ALDEFLUOR kit (StemCell Technologies, British Columbia, Canada) was used to identify the stem/progenitor cells that expressed high levels of the aldehyde dehydrogenase (ALDH) [13]. Briefly, $1 * 10^{6} / \mathrm{ml}$ cells were suspended in Aldefluor Assay Buffer (AAB) and incubated with $5 \mu \mathrm{L}$ ALDH substrate (BAAA) for $45 \mathrm{~min}$. $5 \mu \mathrm{L}$ diethylaminobenzaldehyde (DEAB) was added to a separate sample containing BAAA for an ALDH-inhibited control. Then, samples were washed and resuspended in $\mathrm{AAB}$. Fluorescence-activated cell gates were established using the inhibited control, DEAB, with the fluorescein isothiocyanate (FITC) channel with excitation and emission wavelengths of approximately $495 \mathrm{~nm}$ and $521 \mathrm{~nm}$, respectively. To evaluate the expression of PD-L1 in $\mathrm{ALDH}^{+}$cells, PD-L1 antibody (10F.9G2, GeneTex) and immunoglobulin G (IgG) isotype-matched control (GeneTex) containing BAAA were added to the cells separately. All samples were incubated for 30 minutes at $4^{\circ} \mathrm{C}$. Following incubation, the material was centrifuged, and pellets were resuspended with $500 \mu \mathrm{l}$ assay buffer prior to data acquisition. Flow cytometry analysis was performed on a BD Biosciences FACSCanto, and data analysis was conducted using CellQuest Pro (B\&D Biosciences).

2.3. Tumorsphere Culture. The B16-F0 and B16-F1 melanoma cells were plated as single cells in ultralow attachment six-well plates (Corning, Lowell, MA, USA) and cultured in RPMI 1640 containing $6 \mathrm{mg} / \mathrm{mL}$ glucose (Sigma-Aldrich), $1 \mathrm{mg} / \mathrm{mL}$ NaHCO3 (Sigma-Aldrich), 5 mM HEPES (Sigma-Aldrich), $4 \mu \mathrm{g} / \mathrm{mL}$ heparin (Sigma-Aldrich), $4 \mathrm{mg} / \mathrm{mL}$ bovine serum albumin (Sigma-Aldrich), $20 \mathrm{pg} / \mathrm{mL}$ insulin (Sigma-Aldrich), $\mathrm{N} 2$ supplement (Invitrogen), supplemented with $10 \mathrm{ng} / \mathrm{mL}$ bFGF (Peprotech, Neuilly sur Seine, France), and $20 \mathrm{ng} / \mathrm{mL}$ EGF (Peprotech), as previously described [14]. On the second day after seeding, cells were treated with $10 \mu \mathrm{g}$ anti-PD-L1 (10F.9G2, BioXcell) or control rat immunoglobulin G (IgG). Tumorspheres were observed under microscope 14 days later. Individual spheres with diameters larger than $100 \mu \mathrm{m}$ from each replicate well were visualized and counted with an inverted microscope.
2.4. Assay for Apoptosis. Cells were double-stained with FITC-annexin V and PI according to the manufacturer's instructions (Annexin-V FITC/propidium iodide (PI) Apoptosis Detection Kit; BD Pharmingen). Analysis was performed by flow cytometry. Early apoptotic cells were stained with Annexin-V alone, whereas necrotic and late apoptotic cells were stained with both Annexin-V and PI.

2.5. Animals and Tumor Model. Adult SPF male C57BL/6 mice were implanted subcutaneously on the right flank with either $5 \times 10^{5}$ B16-F0 or $5 \times 10^{5}$ B16-F1 melanoma cells. Then, $100 \mu \mathrm{g}$ anti-PD-L1 or control rat IgG was administered intraperitoneally 3, 6, and 9 days following melanoma cell inoculation. All animals were randomly assigned to two groups of 5 mice each. Tumor size was monitored every two days. All surgical procedures and care given to the animals were in accordance with institutional guidelines.

2.6. Statistical Analysis. All data were reported as the mean \pm standard error. Statistical analysis was performed using GraphPad Prism 5.0 Software (San Diego). A two-tailed paired $t$-test was used to determine significant differences. $P$ values $<0.05$ were considered statistically significant.

\section{Results}

3.1. PD-L1 Expression on ALDH ${ }^{+}$Melanoma Cells. Previous studies have described the isolation of MMICs from mice using ALDEFLUOR/ALDH as a marker [13, 15]. To determine the expression of PD-L1 in MMICs, we detected PD$\mathrm{Ll}^{+} / \mathrm{ALDH}^{+}$subpopulations from these two cell lines. As shown in Figure 1, $\mathrm{ALDH}^{+}$cells were identified in melanoma cell lines by flow cytometry with the ALDEFLUOR kit. Cells were then incubated for $30 \mathrm{~min}$ with mouse monoclonal antibodies specific for PD-L1. The analysis of the percentage of $\mathrm{PD}-\mathrm{Ll}^{+} \mathrm{ALDH}^{+}$cells was gated by $\mathrm{ALDH}^{+}$cells. We found that approximately $10 \%$ to $18 \%$ of the cultured murine B16-F0 cells and B16-F1 cells were $\mathrm{ALDH}^{+}$. Approximately, $5 \%$ of the $\mathrm{ALDH}^{+}$cells were $\mathrm{PD}-\mathrm{Ll}^{+} / \mathrm{ALDH}^{+}$. These data suggest that PD-L1 may be involved in regulating MMICs.

\subsection{PD-L1 Regulated on MMICs Tumorsphere Formation.} To determine whether PD-L1 can mediate MMIC selfrenewal, we cultured melanoma cell lines with anti-PD-L1. The results showed that anti-PD-L1 significantly inhibited tumorsphere formation in B16-F0 and B16-F1 melanoma cells compared to the control groups (Figure 2). Cancer stem cell-derived spheres were dissociated and passaged; they readily formed secondary spheres [16]. Anti- PD-L1 inhibited secondary tumorsphere generation. Anti-PD-L1 induced a 2fold inhibition of tumorsphere formation in B16-F0 cells and approximately 1.4-fold inhibition in B16-F1 melanoma cells, in terms of both number and size, compared with control groups.

3.3. PD-L1 Affected the Apoptosis of MMICs Enriched Cells. Tumorsphere formation has been reported as a measure of the presence of MMICs in enriched cell populations. We further explored the effects of anti-PD-L1 on apoptosis in 

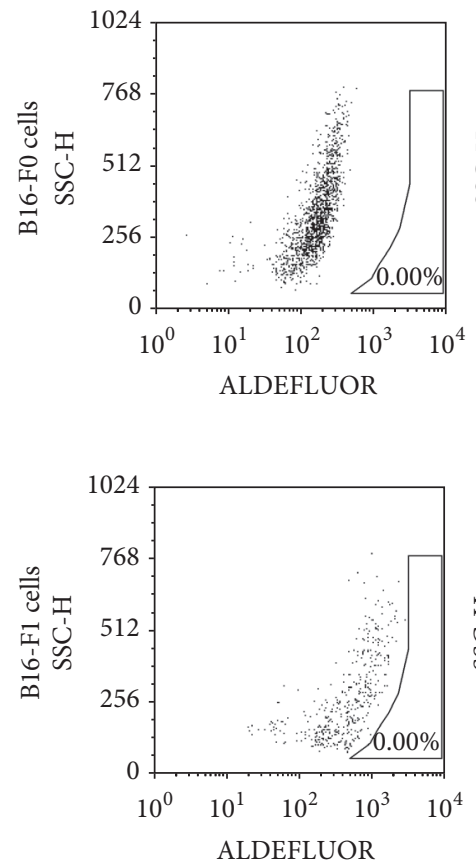

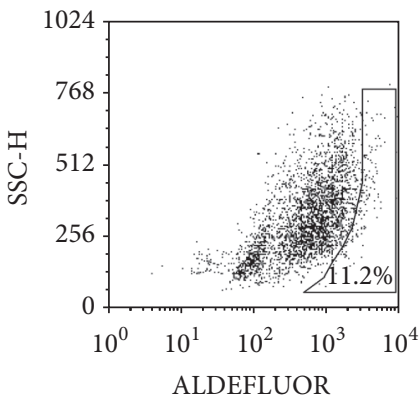

(a)

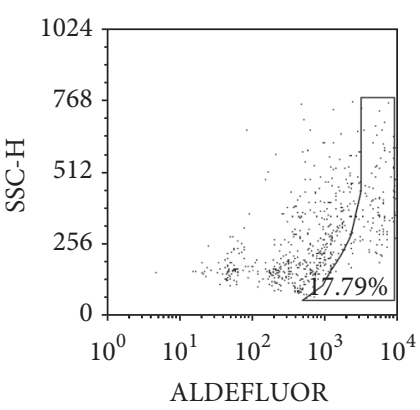

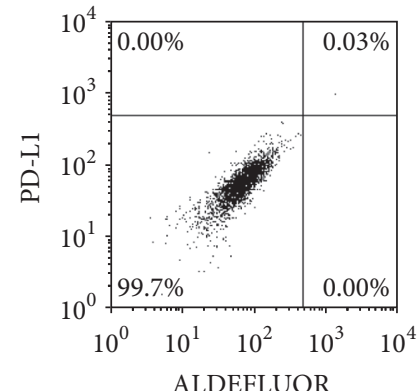
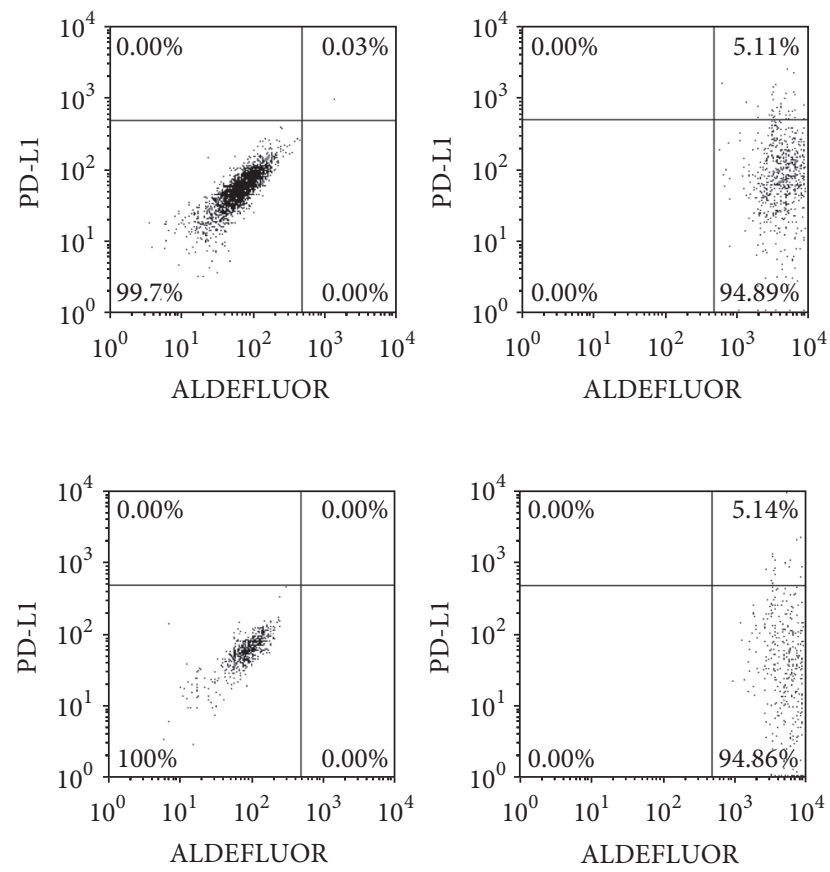

(b)

Figure 1: The expression of PD-L1 on MMICs. (a) The left two scatter plots showed the ALDH ${ }^{+}$cells identified in the B16-F0 melanoma cells by flow cytometry using the ALDEFLUOR kit. Only $\mathrm{ALDH}^{+}$cells were gated for analysis of the percentage of PD-L1 ${ }^{+} \mathrm{ALDH}^{+}$cells. The right two scatter plots showed the percentage of $\mathrm{PD}-\mathrm{Ll}^{+} \mathrm{ALDH}^{+}$cells in B16-F0 melanoma cells. (b) The expression of PD-L1 in ALDH ${ }^{+} \mathrm{B} 16-\mathrm{F} 0$ melanoma cells.

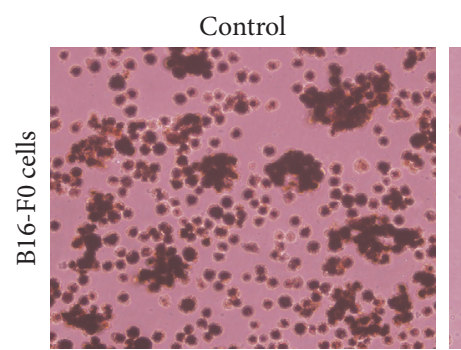

(a)

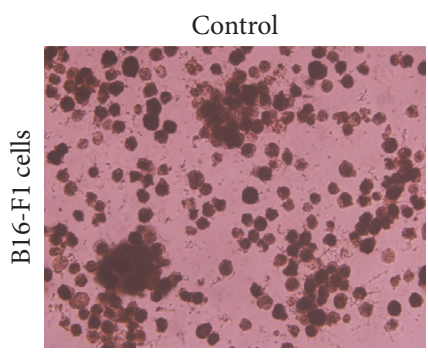

(b)

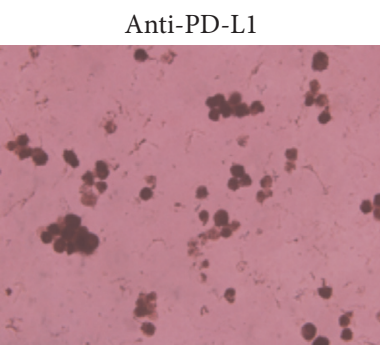

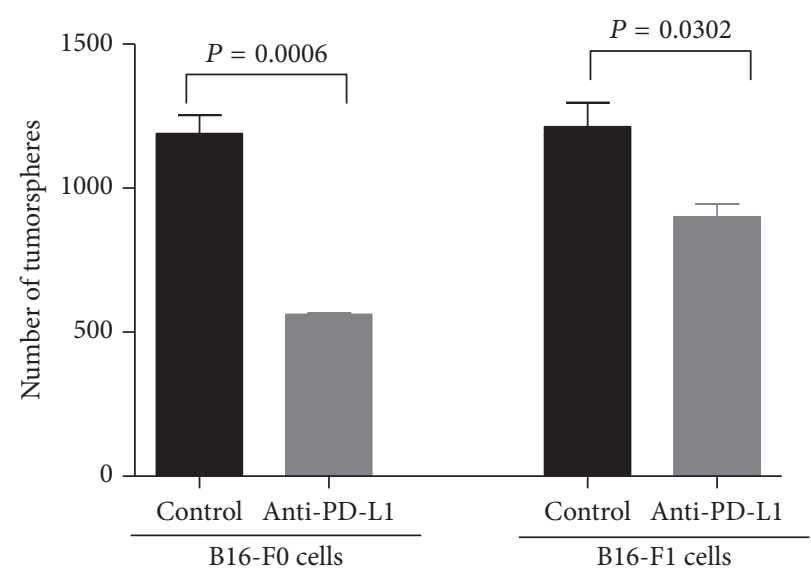

(c)

FIGURE 2: PD-L1 promoted tumorsphere formation. After co-culturing with anti-PD-L1, the sphere formation ability of (a) B16-F0 cells and (b) B16-F1 cells was impaired. (c) The chart showed the number of tumorspheres in each group. Each column represents the mean \pm SE of three independent experiments. 
melanoma tumorspheres. The data illustrated that anti-PDL1 induced significant apoptosis in melanoma tumorspheres (Figure 3). Anti-PD-L1 increased the rate of apoptosis by 2fold in both B16-F0 and B16-F1 tumorspheres. Thus, PD-L1 inhibited apoptosis of MMIC-enriched cells.

3.4. Blockage of PD-L1 Directly Affected MMICs In Vivo. Mice were challenged with melanoma cells (B16-F0 and B16-F1) and treated with $100 \mu \mathrm{g}$ anti-PD-L1 or control rat IgG 3, 6, and 9 days following melanoma cell injection. Other studies have reported that anti-PD-L1 significantly suppressed tumor growth compared with PBS-injected animals in two animal models. Anti-PD-L1 promoted tumor rejection in $50 \%$ of B16-F0 melanoma challenged mice $(P=0.031)$ and $50 \%$ of B16-F1 melanoma challenged mice $(P=0.031$; Figures 4(a) and 4(b)). We observed that anti-PD-L1 decreased residual $\mathrm{ALDH}^{+}$MSCs within the tumor. As shown in Figures 4(c)-4(e), anti-PD-L1 promoted the rejection of 1.5fold residual $\mathrm{ALDH}^{+} \mathrm{MMICs}$ in the B16-F0 animal model $(P=0.016)$ and 1.4 -fold residual $\mathrm{ALDH}^{+} \mathrm{MMICs}$ in the B16-F0 animal model $(P=0.045)$. These results suggest that one mechanism for the anti-tumor effects of anti-PD-L1 is related to its ability to suppress the tumorigenicity capacity of MMICs.

\section{Discussion}

Previous studies have shown that PD-L1 expression is a common phenomenon in immunotherapy-naive melanomas [17-19]. Further studies have indicated that PD-1 expressed by melanoma cells is a tumor growth-promoting mechanism, and PD-1-driven tumorigenesis requires interaction between melanoma-PD-1 and host or melanoma-expressed PD-L1 [18]. Here, we provide several insights into the function of PD-L1 in MMICs, which is separate from its effects on the immune response. Our study found that PD-L1 was expressed in $\mathrm{ALDH}^{+}$MMICs and induced tumorsphere formation. PD-L1 further inhibited the apoptosis of MMIC-enriched cells. Blockage of PD-L1 directly inhibited tumorigenesis in vivo and significantly decreased the residual percentage of MMICs. These results may indicate that melanoma cellintrinsic PD-L1 promotes self-renewal and the tumorigenic capacity of MMICs.

Traditionally, PD-1 ligands have been expressed in tumor cells, leading to T-cell exhaustion and tumor cell evading the immune response, which was thought to require its receptor interaction [20]. Accordingly, several clinical trials have focused on using PD-L1-blocking antibodies to enhance immunity in cancers [21-23]. However, a recent study found that melanoma-PD-1: host-PD-L1 interactions promoted murine melanoma growth [8]. In melanoma, a subpopulation of cells, namely, MMICs, is capable of not only self-renewal, differentiation, plasticity, immune evasion, and multidrug resistance, but also potentially vasculogenic mimicry, and transitioning to migratory and metastasizing derivatives, which are associated with melanoma progression and metastasis [24]. Thus, we believe that melanoma-PD-L1 may contribute to maintaining the stem cell-like properties of MMICs.
MMICs are known to have high ALDH. Previous studies have successfully used ALDH as a marker to isolate MMICs from mice $[13,15]$. Our present flow cytometry results showed that PD-L1 was expressed in $\mathrm{ALDH}^{+}$MMICs. Glioma stem cells expressed lower levels of the PD-L1 than those of differentiated glioma cells, which contributed to the higher sensitivity of glioma stem cells to the cytotoxicity of the IL-2-activated NK cells [25]. In head and neck squamous cell carcinoma, PD-L1 is preferentially expressed in CD $44^{+}$ tumor-initiating cells and inhibits IFN- $\gamma$ secretion by tumorinfiltrating lymphocytes (TILs) incubated with $\mathrm{CD}_{4} 4^{+}$cells [26]. These previous studies focused on the expression of PDL1 in cancer stem cells, which induced immune evasion in cancer. The results presented here demonstrate that anti-PDL1 inhibited the tumorsphere-forming capacity and induced apoptosis in melanoma cancer stem-like cells. Current phaseI studies targeting PD-L1, BMS-936559, and MPDL3280A have reported significant responses and survival benefits [2729]. We showed that anti-PD-L1 inhibited tumor growth in vivo, which is in agreement with these published studies.

It has been established that the expression of PD-L1 is an indicator of poor prognosis for patients' survival in many cancers, such as pulmonary adenocarcinoma, gastric cancer, colorectal cancer, and esophageal cancer [30-33]. In contrast, studies of the prognostic usage of PD-L1 expression are inconsistent in melanoma [34]. As McLaughlin et al. [35] demonstrated in NSCLC, several factors contributed to false-negative PD-L1 findings, including the fact that tumor samples may be inadequate or not representative of the entire tumor mass, different anti-PD-L1-directed antibodies perform differently, and a quantitative interpretation of immunohistochemical stains has some deficiencies. As described above, PD-L1 expression in melanoma cells showed marked heterogeneity, which may have implications on the study of the prognostic usage of PD-L1 expression analysis. The present experimental data showed that anti-PD-L1 significantly decreased the residual percentage of MMICs, which indicates that the melanoma-PD-L1 pathway may be one of the many mechanisms involved in PD-L1-mediated melanoma progression. According to Tamai et al. [12], PDL1 can directly affect cancer stem cells, which is distinct from its immunomodulatory action.

Taken together, our results suggest that melanoma-PDL1 can enhance tumorigenesis by maintaining the stem cell properties in MMICs. Future studies are needed to elucidate the underlying cellular and molecular mechanisms, which will be helpful to maximize its clinical benefits.

\section{Conflicts of Interest}

No conflicts of interest exit in the submission of this manuscript.

\section{Acknowledgments}

The financial supports of this work are received from the National Nature Sciences Foundation of China (no. 81301954) and Hubei Provincial Health and Family Planning Scientific 

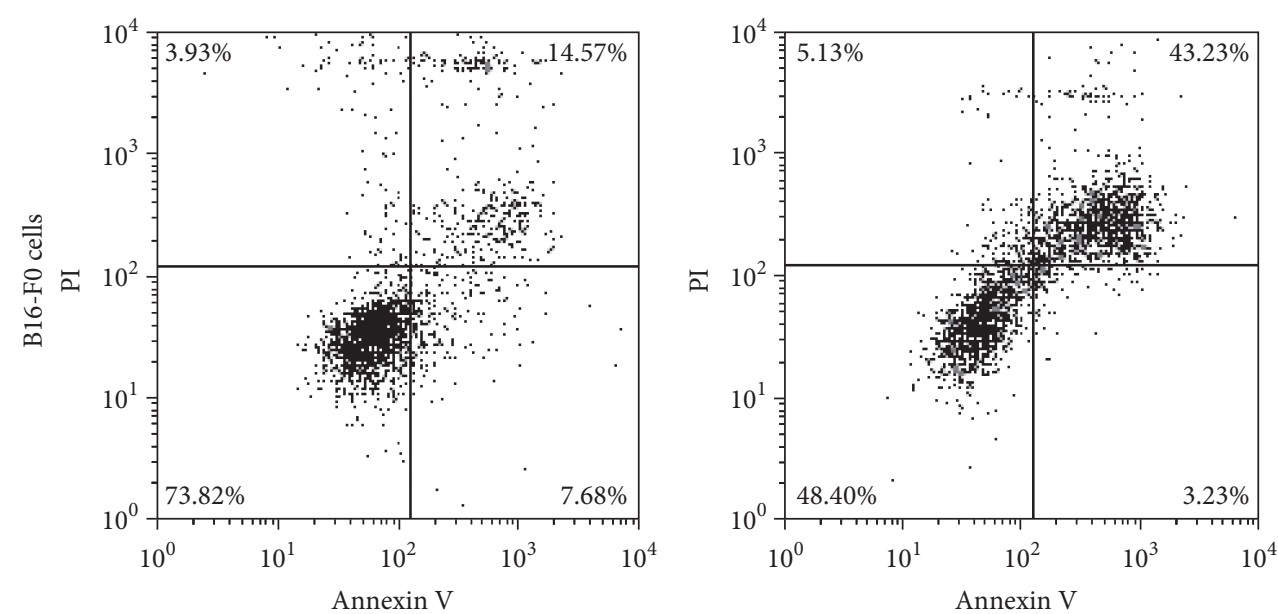

(a)
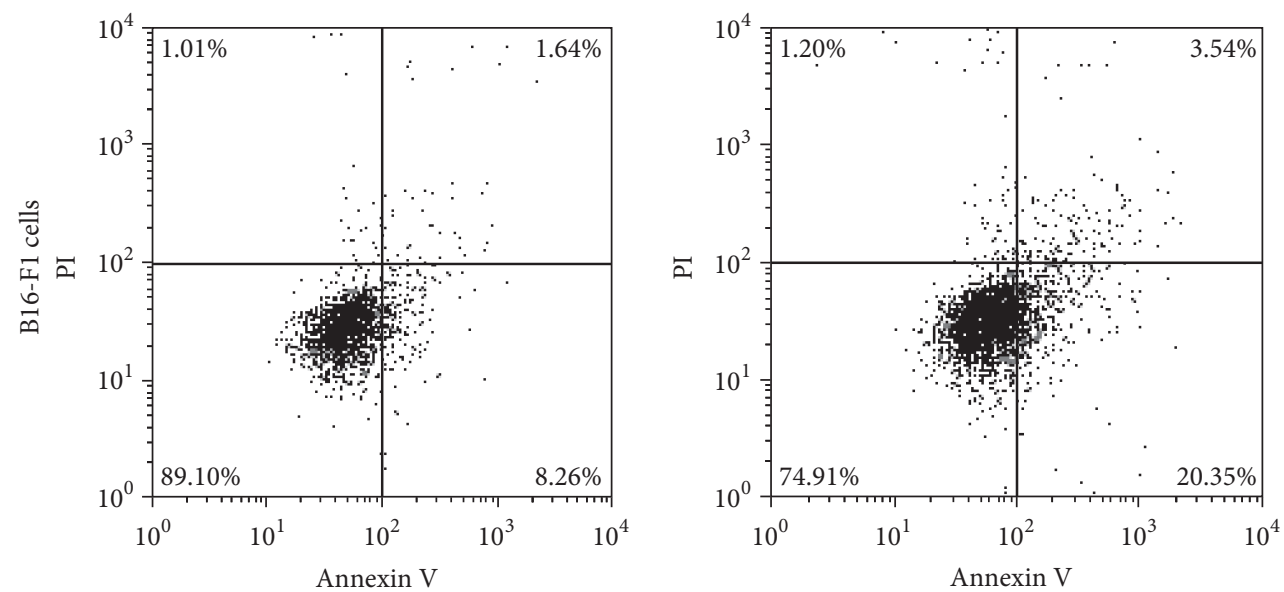

(b)

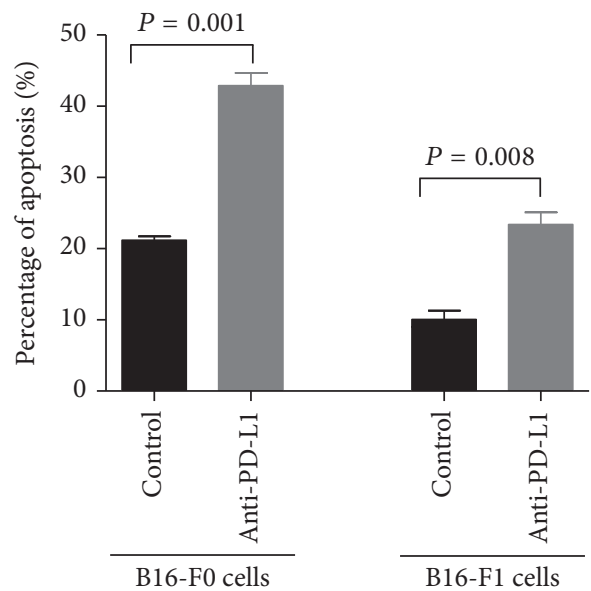

(c)

FIGURE 3: PD-L1 inhibited the apoptosis of sphere cells. After coculturing with anti-PD-L1 for 14 days, tumorspheres were collected and then dissociated into a single cell suspension. The apoptosis rates of (a) B16-F0 spheres and(b) B16-F1 spheres were measured using flow cytometry. (c) The chart shows the apoptosis rate in each group. Each column represents the mean \pm SE of three independent experiments. 


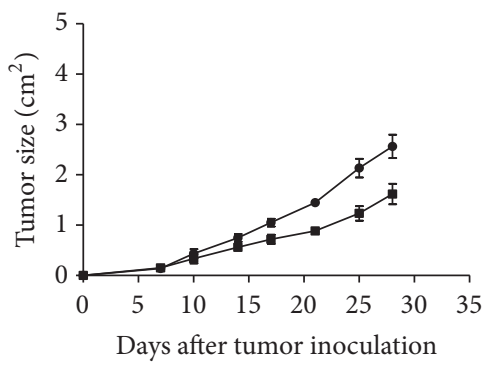

$\rightarrow$ Control

- Anti-PD-L1

(a)

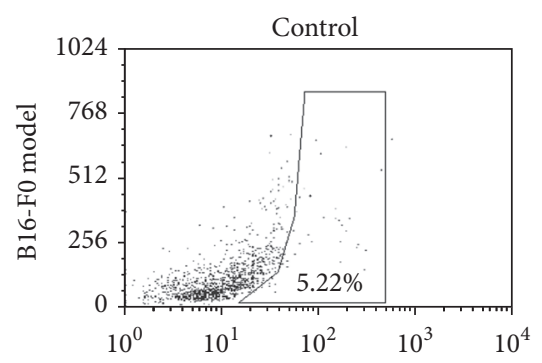

(c)

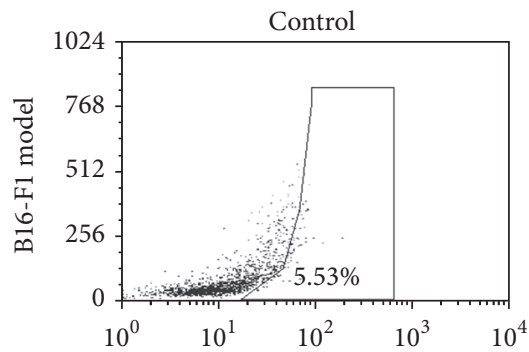

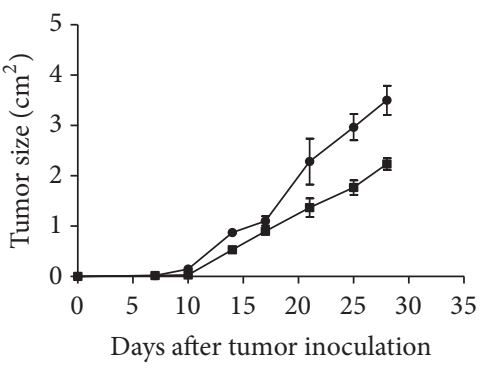

$\rightarrow$ Control

- Anti-PD-L1

(b)
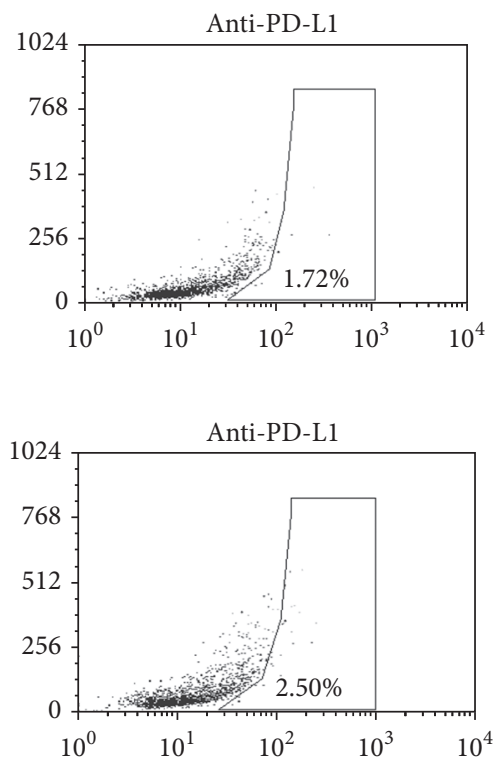

(d)

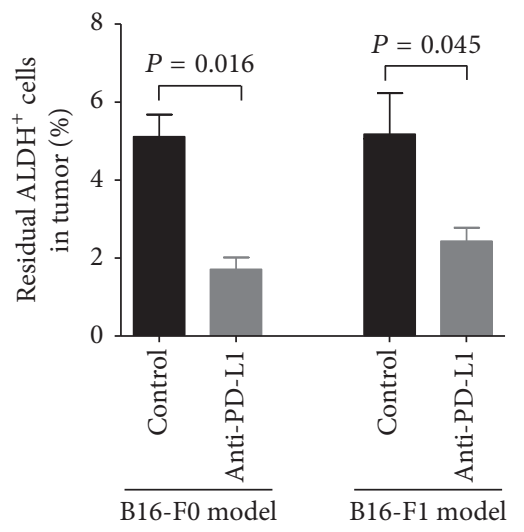

(e)

FIGURE 4: Blockage of PD-L1 affects MMICs in vivo. C57BL/6 mice were separately inoculated with (a) B16-F0 cells and(b) B16-F1 cells. Then, mice were administered anti-PD-L1 3, 6, and 9 days after melanoma cell injection. Tumor growth was monitored. The results are shown as the mean \pm SE of five mice in each group. (c) Images of tumors from representative animals used in (a) and (b). At the end of the experiment, residual $\mathrm{ALDH}^{+}$MMICs within the tumor were analyzed by flow cytometry in the (c) B16-F0 and B16-F1 (d) cell models. (e) The data for residual $\mathrm{ALDH}^{+} \mathrm{MMICs}$ within two tumor models are shown as the mean $\pm \mathrm{SE}$ of five mice in each group. 
Research project (no. 2015060101010043). We appreciate the English Language Service (Ameican Journal Experts) who edit this manuscript.

\section{References}

[1] W. Zou, J. D. Wolchok, and L. Chen, "PD-L1 (B7-H1) and PD1 pathway blockade for cancer therapy: mechanisms, response biomarkers, and combinations," Science Translational Medicine, vol. 8, no. 328, Article ID 328rv4, 2016.

[2] G. F. Murphy, B. J. Wilson, S. D. Girouard, N. Y. Frank, and M. H. Frank, "Stem cells and targeted approaches to melanoma cure," Molecular Aspects of Medicine, vol. 39, pp. 33-49, 2014.

[3] T. Schatton, U. Schütte, N. Y. Frank et al., "Modulation of Tcell activation by malignant melanoma initiating cells," Cancer Research, vol. 70, no. 2, pp. 697-708, 2010.

[4] H. Pandha and G. Pawelec, "Immune checkpoint targeting as anti-cancer immunotherapy: promises, questions, challenges and the need for predictive biomarkers at ASCO 2015," Cancer Immunology, Immunotherapy, vol. 64, no. 9, pp. 1071-1074, 2015.

[5] P. Sharma and J. P. Allison, "Immune checkpoint targeting in cancer therapy: toward combination strategies with curative potential," Cell, vol. 161, no. 2, pp. 205-214, 2015.

[6] J. Duraiswamy, K. M. Kaluza, G. J. Freeman, and G. Coukos, "Dual blockade of PD-1 and CTLA-4 combined with tumor vaccine effectively restores T-cell rejection function in tumors," Cancer Research, vol. 73, no. 12, pp. 3591-3603, 2013.

[7] M. K. Callahan, M. A. Postow, and J. D. Wolchok, "CTLA-4 and PD-1 pathway blockade: combinations in the clinic," Frontiers in Oncology, vol. 4, p. 385, 2014.

[8] S. Kleffel, C. Posch, S. R. Barthel et al., "Melanoma cell-intrinsic PD-1 receptor functions promote tumor growth," Cell, vol. 162, no. 6, pp. 1242-1256, 2015.

[9] H. Ghebeh, A. Tulbah, S. Mohammed et al., "Expression of B7-H1 in breast cancer patients is strongly associated with high proliferative Ki-67-expressing tumor cells," International Journal of Cancer, vol. 121, no. 4, pp. 751-758, 2007.

[10] Y. Yao, R. Tao, X. Wang, Y. Wang, Y. Mao, and L. F. Zhou, "B7-H1 is correlated with malignancy-grade gliomas but is not expressed exclusively on tumor stem-like cells," NeuroOncology, vol. 11, no. 6, pp. 757-766, 2009.

[11] Y. Lee, J. H. Shin, M. Longmire et al., "Cd $44^{+}$cells in head and neck squamous cell carcinoma suppress t-cell-mediated immunity by selective constitutive and inducible expression of PD-L1," Clinical Cancer Research, vol. 22, no. 14, pp. 3571-3581, 2016.

[12] K. Tamai, M. Nakamura, M. Mizuma et al., "Suppressive expression of CD274 increases tumorigenesis and cancer stem cell phenotypes in cholangiocarcinoma," Cancer Science, vol. 105, no. 6, pp. 667-674, 2014.

[13] N. Ning, Q. Pan, F. Zheng et al., "Cancer stem cell vaccination confers significant antitumor immunity," Cancer Research, vol. 72, no. 7, pp. 1853-1864, 2012.

[14] A.-R. Choi, J.-R. Park, R.-J. Kim et al., "Inhibition of Wnt1 expression reduces the enrichment of cancer stem cells in a mouse model of breast cancer," Biochemical and Biophysical Research Communications, vol. 425, no. 2, pp. 436-442, 2012.

[15] Y. Luo, K. Dallaglio, Y. Chen et al., "ALDH1A isozymes are markers of human melanoma stem cells and potential therapeutic targets," Stem Cells, vol. 30, no. 10, pp. 2100-2113, 2012.
[16] M. F. Clarke, J. E. Dick, P. B. Dirks et al., "Cancer stem cellsperspectives on current status and future directions: AACR workshop on cancer stem cells," Cancer Research, vol. 66, no. 19, pp. 9339-9344, 2006.

[17] J. Gadiot, A. I. Hooijkaas, A. D. M. Kaiser, H. Van Tinteren, H. Van Boven, and C. Blank, "Overall survival and PD-L1 expression in metastasized malignant melanoma," Cancer, vol. 117, no. 10, pp. 2192-2201, 2011.

[18] J. M. Taube, R. A. Anders, G. D. Young et al., "Colocalization of inflammatory response with B7-H1 expression in human melanocytic lesions supports an adaptive resistance mechanism of immune escape," Science Translational Medicine, vol. 4, no. 127, Article ID 127ra37, 2012.

[19] R. Hino, K. Kabashima, Y. Kato et al., "Tumor cell expression of programmed cell death-1 ligand 1 is a prognostic factor for malignant melanoma," Cancer, vol. 116, no. 7, pp. 1757-1766, 2010.

[20] J. M. Taube, A. Klein, J. R. Brahmer et al., "Association of PD-1, PD-1 ligands, and other features of the tumor immune microenvironment with response to anti-PD-1 therapy," Clinical Cancer Research, vol. 20, no. 19, pp. 5064-5074, 2014.

[21] J. Larkin, V. Chiarion-Sileni, R. Gonzalez et al., "Combined nivolumab and ipilimumab or monotherapy in untreated Melanoma," The New England Journal of Medicine, vol. 373, no. 1, pp. 23-34, 2015.

[22] M. A. Postow, J. Chesney, A. C. Pavlick et al., "Nivolumab and ipilimumab versus ipilimumab in untreated melanoma," The New England Journal of Medicine, vol. 372, pp. 2006-2017, 2015.

[23] P. C. Tumeh, C. L. Harview, J. H. Yearley et al., "PD-1 blockade induces responses by inhibiting adaptive immune resistance," Nature, vol. 515, no. 7528, pp. 568-571, 2014.

[24] G. Parmiani, "Melanoma cancer stem cells: markers and functions," Cancers, vol. 8, no. 3, article no. 34, 2016.

[25] B. Y. Huang, Y. P. Zhan, W. J. Zong et al., "The PD-1/B7-H1 pathway modulates the natural killer cells versus mouse glioma stem cells," PLoS ONE, vol. 10, no. 8, Article ID e0134715, 2015.

[26] Y. Lee and J. Sunwoo, "PD-L1 is preferentially expressed on CD44+ tumor-initiating cells in head and neck squamous cell carcinoma," Journal for Immuno Therapy of Cancer, vol. 2, no. 1, p. 1, 2013.

[27] J. R. Brahmer, S. S. Tykodi, L. Q. M. Chow et al., "Safety and activity of anti-PD-L1 antibody in patients with advanced cancer," The New England Journal of Medicine, vol. 366, no. 26, pp. 2455-2465, 2012.

[28] O. Hamid and R. D. Carvajal, "Anti-programmed death-1 and anti-programmed death-ligand 1 antibodies in cancer therapy," Expert Opinion on Biological Therapy, vol. 13, no. 6, pp. 847-861, 2013.

[29] O. Hamid, C. Robert, A. Daud et al., "Safety and tumor responses with lambrolizumab (anti-PD-1) in melanoma," The New England Journal of Medicine, vol. 369, no. 2, pp. 134-144, 2013.

[30] C.-Y. Yang, M.-W. Lin, Y.-L. Chang, C.-T. Wu, and P.-C. Yang, "Programmed cell death-ligand 1 expression in surgically resected stage i pulmonary adenocarcinoma and its correlation with driver mutations and clinical outcomes," European Journal of Cancer, vol. 50, no. 7, pp. 1361-1369, 2014.

[31] R. A. Droeser, C. Hirt, C. T. Viehl et al., "Clinical impact of programmed cell death ligand 1 expression in colorectal cancer," European Journal of Cancer, vol. 49, no. 9, pp. 2233-2242, 2013. 
[32] L. Chen, H. Deng, M. Lu, B. Xu, Q. Wang, J. Jiang et al., "B7-H1 expression associates with tumor invasion and predicts patient's survival in human esophageal cancer," International Journal of Clinical and Experimental Pathology, vol. 7, pp. 6015-6023, 2014.

[33] Y. Qing, Q. Li, T. Ren et al., "Upregulation of PD-L1 and APE1 is associated with tumorigenesis and poor prognosis of gastric cancer," Drug Design, Development and Therapy, vol. 9, pp. 901909, 2015.

[34] J. Madore, R. E. Vilain, A. M. Menzies, H. Kakavand, J. S. Wilmott, J. Hyman et al., "PD-L1 expression in melanoma shows marked heterogeneity within and between patients: Implications for anti-PD-1/PD-L1 clinical trials," Pigment Cell and Melanoma Research, vol. 28, no. 3, pp. 245-253, 2015.

[35] J. McLaughlin, G. Han, K. A. Schalper et al., "Quantitative assessment of the heterogeneity of PD-L1 expression in nonsmall-cell lung cancer," JAMA Oncology, vol. 2, no. 1, pp. 46-54, 2016. 


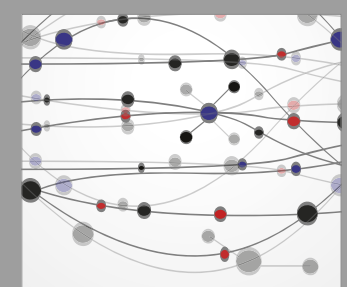

The Scientific World Journal
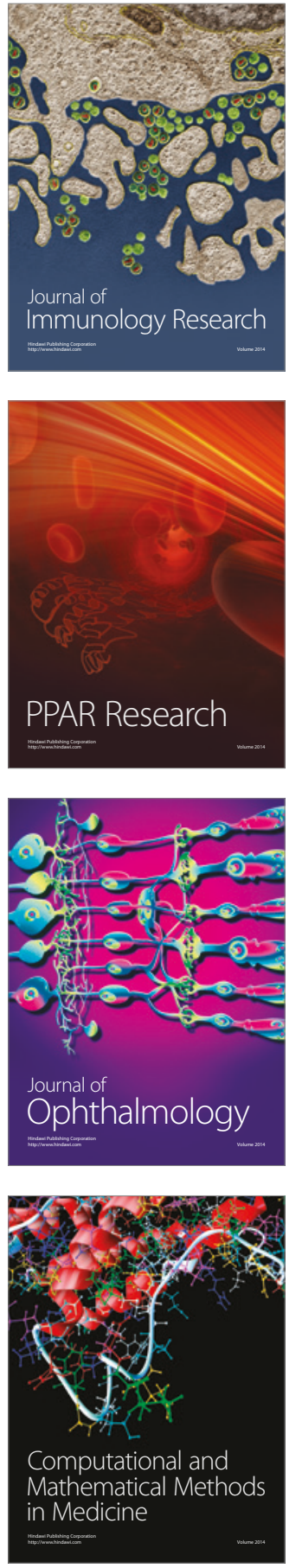

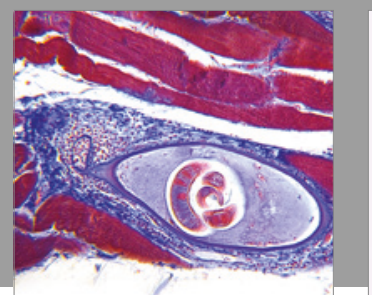

Gastroenterology Research and Practice
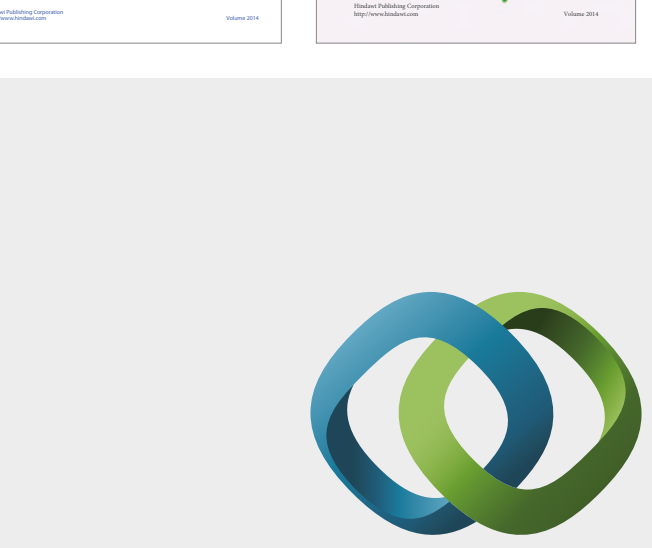

\section{Hindawi}

Submit your manuscripts at

https://www.hindawi.com
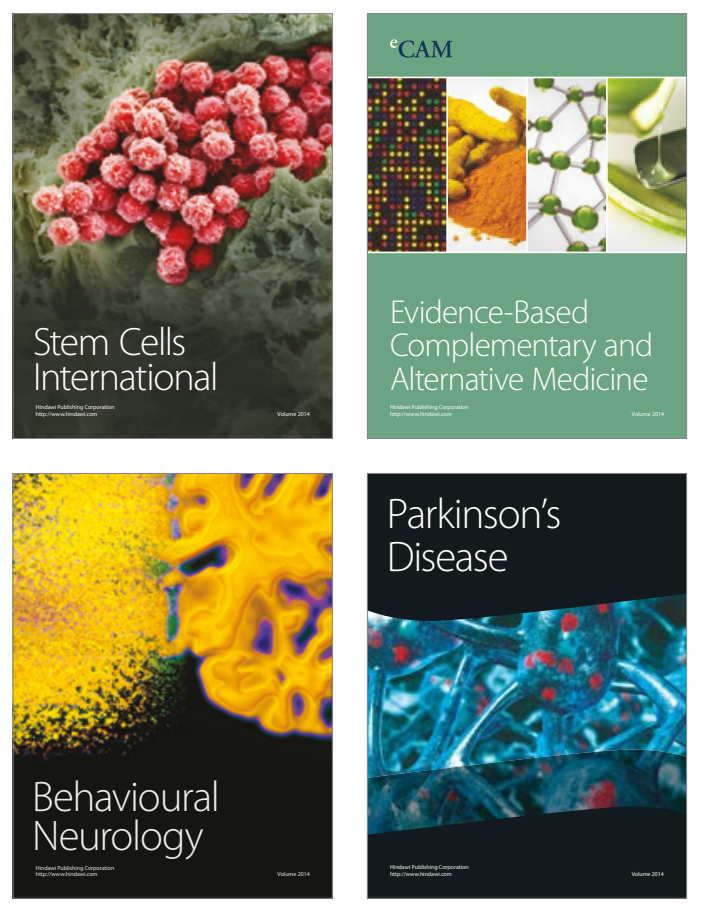
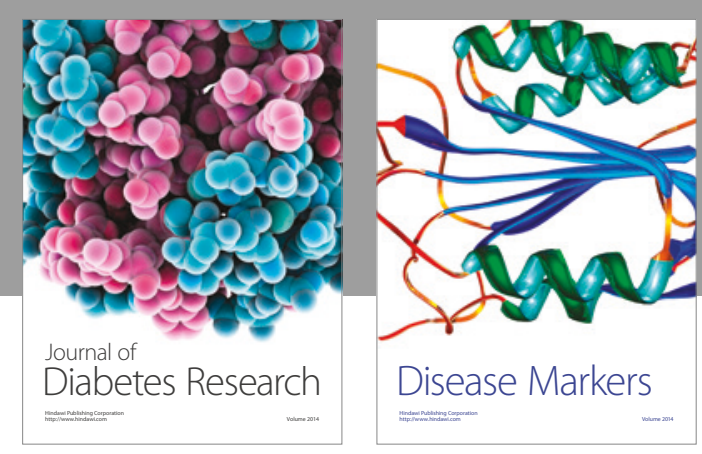

Disease Markers
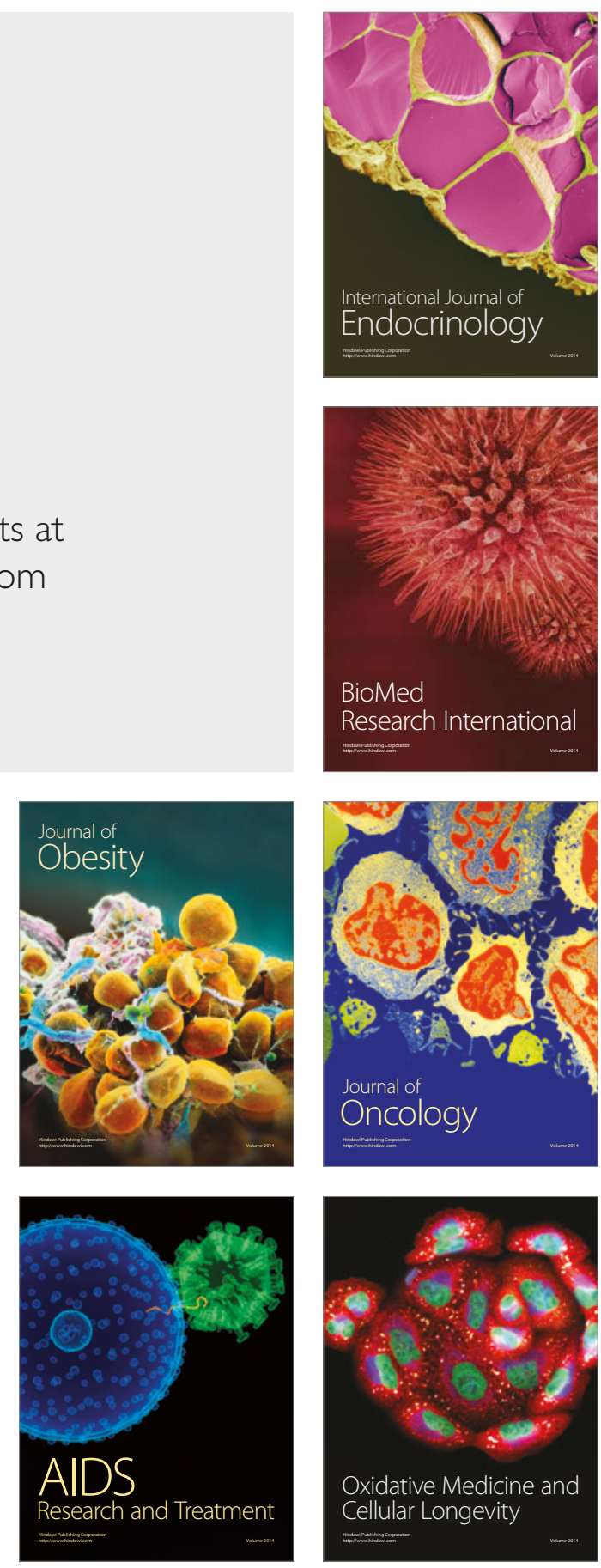